\title{
Article \\ Personalized Medicine Based on Nasal Epithelial Cells: Comparative Studies with Rectal Biopsies and Intestinal Organoids
}

\author{
Iris A. L. Silva (D), Violeta Railean, Aires Duarte and Margarida D. Amaral * \\ BioISI-Biosystems and Integrative Sciences Institute, Faculty of Sciences, University of Lisboa, \\ 1749-016 Lisboa, Portugal; iasilva@fc.ul.pt (I.A.L.S.); vrailean@fc.ul.pt (V.R.); amduarte@fc.ul.pt (A.D.) \\ * Correspondence: msamaral@fc.ul.pt
}

check for

updates

Citation: Silva, I.A.L.; Railean, V.;

Duarte, A.; Amaral, M.D.

Personalized Medicine Based on

Nasal Epithelial Cells: Comparative

Studies with Rectal Biopsies and

Intestinal Organoids. J. Pers. Med.

2021, 11, 421. https://doi.org/

10.3390/jpm11050421

Academic Editors: Cornelis K. van der Ent and Harry G. M. Heijerman

Received: 24 April 2021

Accepted: 12 May 2021

Published: 16 May 2021

Publisher's Note: MDPI stays neutral with regard to jurisdictional claims in published maps and institutional affiliations.

Copyright: (C) 2021 by the authors Licensee MDPI, Basel, Switzerland. This article is an open access article distributed under the terms and conditions of the Creative Commons Attribution (CC BY) license (https:/ / creativecommons.org/licenses/by/ $4.0 /)$.

\begin{abstract}
As highly effective CFTR modulator therapies (HEMT) emerge, there is an unmet need to find effective drugs for people with $\mathrm{CF}(\mathrm{PwCF})$ with ultra-rare mutations who are too few for classical clinical trials and for whom there are no drug discovery programs. Therefore, biomarkers reliably predicting the benefit from CFTR modulator therapies are essential to find effective drugs for PwCF through personalized approaches termed theranostics. Here, we assess CFTR basal function and the individual responses to CFTR modulators in primary human nasal epithelial (pHNE) cells from PwCF carrying rare mutations and compare these measurements with those in native rectal biopsies and intestinal organoids, respectively, in the same individual. The basal function in pHNEs shows good correlation with CFTR basal function in rectal biopsies. In parallel, CFTR rescue in pHNEs by CFTR modulators correlates to that in intestinal organoids. Altogether, results show that $\mathrm{pHNEs}$ are a bona fide theranostic model to assess CFTR rescue by CFTR modulator drugs, in particular for PwCF and rare mutations.
\end{abstract}

Keywords: CFTR; human nasal epithelial cells; rectal biopsies; intestinal organoids; CFTR modulators; theranostic

\section{Introduction}

Cystic fibrosis (CF) is the most common lethal genetic disorder, caused by mutations in the gene encoding for Cystic Fibrosis Transmembrane Conductance Regulator (CFTR), a channel responsible for chloride $\left(\mathrm{Cl}^{-}\right)$and bicarbonate $\left(\mathrm{HCO}_{3}{ }^{-}\right)$transport in epithelial cells [1,2]. Dysfunctional CFTR leads to the disruption of salts and fluid homeostasis in the cells of the pancreas, airways and intestine, among others [3]. Although CF is a multi-organ disease, progressive loss of lung function is the major cause of morbidity and lethality [4]. In the airways, functional failure of CFTR results in the accumulation of thick and sticky mucus that impedes mucociliary clearance, contributing to chronic bacterial infections and persistent inflammation [5,6].

More than 2100 mutations have been reported so far in the CFTR gene [7]. F508del is the most frequent mutation occurring in $\sim 80 \%$ of all individuals with CF worldwide and is characterized by defective CFTR processing and trafficking [1]. Currently available CFTR modulators that target the underlying CFTR defect are approved for the treatment of individuals carrying specific CFTR mutations, including F508del [8,9]. However, $18 \%$ of individuals with CF worldwide are not eligible for these therapies since they lack F508del or any other mutation targeted by currently approved CFTR modulator drugs [10]. Moreover, the traditional randomized clinical trials requiring large numbers of individuals to demonstrate drug efficacy become impracticable for those with rare mutations [11,12]. In addition, besides the CFTR genotype, several factors, including modifier genes, environmental factors and lifestyle, are known to modulate the CF disease prognosis and drug response, which highlights the importance of personalized therapy [13-15]. 
Recently, different cellular models have been developed to determine the response of CFTR mutants to modulators through a personalized CFTR pharmacotherapy approach [11]. The most common cellular models include primary human nasal (pHNE) and bronchial (pHBE) epithelial cells and intestinal organoids obtained from rectal biopsies [16-27]. Although pHBEs are the gold standard to test for CFTR modulator efficacy since they can recapitulate the in vivo morphology and key processes happening in lungs [28], intestinal organoids are the most advanced model in $\mathrm{CF}$ research, incorporating many physiological relevant tissue features [29]. Moreover, the measurement of CFTR-mediated $\mathrm{Cl}^{-}$secretion in human rectal biopsies has been established as a sensitive and robust biomarker for $\mathrm{CF}$ diagnosis and prognosis [30-34].

In this work, we aimed to compare CFTR basal function in pHNEs and rectal biopsies from individuals carrying rare mutations, namely P205S, D614G, N1303K, G85E and F508del, and assess whether $\mathrm{pHNEs}$ reflect the measurements performed in rectal biopsies in the same individual. Moreover, we also aimed to compare modulator responses in pHNEs and in intestinal organoids also from the same individuals.

Our results show that data in pHNEs significantly correlate with those in both cellular systems, demonstrating that pHNEs are good models both to assess CFTR basal activity and to predict the therapeutic efficacy of CFTR modulator drugs.

\section{Materials and Methods}

\subsection{Cohort and Ethical Approval}

All specimens were obtained from one healthy control and six individuals with $\mathrm{CF}$ (carrying F508del/F508del, P205S/Y1092X (two twin sisters), F508del/D614G, F508del/N1303K and G85E/1717-2G>A CFTR genotypes, Table 1) after receiving patient written consent. The study was conducted in accordance with the Declaration of Helsinki, and the protocol was approved by the Ethics Committee of the Santa Maria Hospital and D. Estefânia Hospital (Lisboa, Portugal).

Table 1. Individuals that participated in the study and their respective genotypes.

\begin{tabular}{cc}
\hline Individual Code & Genotype \\
\hline 1 & WT \\
2 & F508del/F508del \\
3 & P205S/Y1092X \\
4 & D614G/F508del \\
5 & N1303K/F508del \\
6 & G85E/1717-1G $>$ A \\
7 & P205S/Y1092X \\
\hline
\end{tabular}

\subsection{Rectal Biopsies}

Human rectal biopsies were obtained by rectoscopy and forceps biopsy as described previously $[30,35,36]$. The tissue was mounted on a circulating micro-Ussing chamber as described previously $[32,33]$. The transepithelial measurements were performed under open-circuit conditions in a continuously perfused micro-Ussing chamber at $37^{\circ} \mathrm{C}$, as described previously $[30,32,33]$. Briefly, the rectal epithelium was continuously perfused ( $5 \mathrm{~mL} / \mathrm{min}$ ) with Ringer solution with the following composition (mmol/L): $\mathrm{NaCl} 145$, KH2PO4 0.4, K2HPO4 1.6, D-glucose 5, $\mathrm{MgCl}_{2}$ 1, Ca-gluconate 1.3, pH 7.40. Tissues were equilibrated in the micro-Ussing chambers for $30 \mathrm{~min}$ in perfused Ringer solution before measurements. Amiloride (Amil, $20 \mu \mathrm{M}$, luminal) was added to block electrogenic sodium $(\mathrm{Na}+$ ) absorption through the epithelial $\mathrm{Na}+$ channel $(\mathrm{ENaC})$. Indomethacin (Indo, $10 \mu \mathrm{M}$, basolateral) was applied for 20-40 min to inhibit endogenous cAMP formation through prostaglandins. cAMP-dependent and cholinergic $\mathrm{Cl}^{-}$secretion in human rectal tissues relies on functional CFTR. Thus, we used 3-isobutyl-1-methylxantine (IBMX/I, $100 \mu \mathrm{M}$, basolateral) and forskolin (Fsk, $2 \mu \mathrm{M}$, basolateral) to activate cAMP-dependent 
$\mathrm{Cl}^{-}$secretion and carbachol (CCH, $100 \mu \mathrm{M}$, basolateral) for cholinergic co-activation. In this protocol, Amil was constantly perfused in the luminal side during the experiment.

The parameter that was recently published as being better correlated with disease diagnosis and prognosis was the voltage (Vte) of the maximum activation of CFTR that corresponds to the sum of the IBMX/Fsk and IBMX/Fsk/CCH responses. The percentage

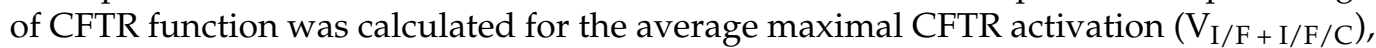
normalized to the corresponding mean value determined previously for a reference non- $\mathrm{CF}$ control group [30].

\subsection{Primary Airway Cell Cultures}

Primary human nasal epithelial cells (pHNEs) were obtained from nasal brushing as before [37]. After expansion, cells were seeded on porous membranes and cultured under air-liquid interface (ALI) conditions for 21 days following protocols by Jeffrey Beekman's lab (Utrecht, The Netherlands, submitted to Cell Rep Med). Differentiated monolayers of pHNEs were incubated with $3 \mu \mathrm{M}$ VX809, $5 \mu \mathrm{M}$ VX661 (Selleckchem, TX, USA), or DMSO alone for $48 \mathrm{~h}$ prior to measurements in an Ussing Chamber. The pHNE monolayers were mounted on micro-Ussing chambers and analyzed under opencircuit conditions, as described previously [16]. CFTR was stimulated by the presence of forskolin $(2 \mu \mathrm{M})+$ IBMX $(100 \mu \mathrm{M})$, and specificity was evaluated after using CFTR inhibitor $172(25 \mu \mathrm{M})$. Experiments were performed in the presence of amiloride $(20 \mu \mathrm{M})$ to inhibit the Epithelial Sodium $(\mathrm{Na}+)$ Channel $(\mathrm{ENaC})$ and thus avoid interference of $\mathrm{ENaC}$ mediated currents. In pHNEs, the maximum CFTR activation corresponds to the sum of IBMX/Fsk and IBMX/Fsk+VX-770 responses [16]. CFTR rescue by CFTR modulators in pHNEs was assessed by comparison of the maximum activation of CFTR between control pHNE (DMSO) and treated pHNE (VX809 or VX661). The individuals were considered responders when this difference was statistically different $(p$-value $<0.05)$. Each experiment was performed at least three times.

\subsection{Ussing Chamber Measurements}

Transepithelial voltage (Vte) values were continuously recorded using Power Lab software (AD Instruments Inc., Dunedin, New Zealand) and referenced to the basal surface of the epithelium. Transepithelial resistance (Rte) was determined by applying short (1 s) intermittent current pulses $(0.5 \mu \mathrm{A})$. The equivalent short-circuit currents $(\Delta \mathrm{Ieq}$-sc) were calculated according to Ohm's law from Vte and Rte $(\Delta \mathrm{Ieq}-\mathrm{sc}=\mathrm{Vte} / \mathrm{Rte})$, with appropriate correction for fluid resistance [31].

\subsection{Human Intestinal Organoids Culture}

Intestinal organoids were obtained by crypt isolation from rectal biopsies and seeded in 50\% Matrigel as described by Vonk et al. [36]. The organoids were passaged $\sim 1$ per week, and the medium was refreshed every 2-3 days.

\subsection{Forskolin-Induced Swelling (FIS) Assay}

Forskolin-induced swelling assay was adapted from Dekkers et al. [18]. Briefly, rectal organoids were seeded in a flat-bottom 96-well culture plate in $4 \mu \mathrm{L}$ of Matrigel and immersed in $50 \mu \mathrm{L}$ of culture medium, with or without $3 \mu \mathrm{M} \mathrm{VX809} \mathrm{(lumacaftor)} \mathrm{or}$ $5 \mu \mathrm{M}$ VX661 (tezacaftor). After $24 \mathrm{~h}$, the organoids were incubated with $3 \mu \mathrm{M}$ calcein green (Invitrogen, Waltham, MA, USA) for $20 \mathrm{~min}$; before imaging, they were stimulated with different concentrations of forskolin $(0.02,0.128,0.8$, and $5 \mu \mathrm{M})$ (Sigma-Aldrich, St. Louis, MO, USA) alone or in combination with $3 \mu \mathrm{M}$ VX770 (ivacaftor, Selleckchem, Houston, TX, USA). The organoids were directly analyzed by confocal live cell microscopy (Leica SP8, Leica Microsystems, Wetzlar, Germany) at $37^{\circ} \mathrm{C}$ with $5 \% \mathrm{CO}_{2}$ for $60 \mathrm{~min}$ and images were acquired every $10 \mathrm{~min}$. Forskolin-induced organoid swelling was quantified using Cell Profiler software (Broad Institute's Imaging Platform, Cambridge, MA, USA), image processing and lab-designed scripts [38]. GraphPad Prism was used to obtain and 
plot values for organoid surface area. Organoid swelling analysis is expressed as the absolute area under the curve (AUC) calculated from the normalized surface area increase (baseline $=100 \%, t=60 \mathrm{~min}$ ). Quantification of CFTR response to modulators in organoids was calculated as the difference between non-treated organoids (Fsk) and treated organoids (VX809 or VX661). The individuals were considered "responders" when the AUC was above 1000 after CFTR modulators incubation, as described in Dekkers et al. [18] and Ramalho et al. [39]. Each experiment was performed at least three times and each condition had technical duplicates within the same experiment.

\subsection{Statistical Analyses}

Statistical analyses were performed using one-way ANOVA on GraphPad Prism8 software, and $p$-values of $<0.05$ were considered significant.

\section{Results}

\subsection{CFTR Basal Function in Primary Human Nasal Epithelial ( $p H N E$ ) Cells Correlates with the} One Measured in Rectal Biopsies

In this work, we determined the correlation between CFTR-mediated $\mathrm{Cl}^{-}$secretion measured in pHNEs and in rectal biopsies. Our results show that in non-CF individuals (controls), Fsk induced a lumen-negative deflection which corresponds to the activation of normal CFTR, both in pHNEs and rectal biopsies (Figure 1A,B, individual 1). Additionally, as expected, in samples from a person with $\mathrm{CF}$ (PwCF) homozygous for F508del, Fsk failed to induce any CFTR-mediated $\mathrm{Cl}^{-}$secretion in both pHNEs and rectal biopsies (Figure 1C,D, individual 2). By analyzing samples from PwCF with four different rare mutations-P205S, D614G, N1303K, and G85E-we were able to observe that residual CFTR-mediated $\mathrm{Cl}^{-}$secretion was detected both in pHNEs and rectal biopsies from PwCF 3 and 4 with P205S/Y1092X and D614G/F508del genotypes, respectively (Figure 1E-H, individuals 3 and 4). In pHNEs and rectal biopsies from individuals 5 and 6 (Figure 1I-L, individuals 5 and 6) with N1303K/F508del and G85E/1717-1G>A genotypes, respectively, there was a total lack of CFTR-mediated $\mathrm{Cl}^{-}$secretion.

We then performed a comparative analysis of CFTR-mediated $\mathrm{Cl}^{-}$secretion in $\mathrm{pHNEs}$ and rectal biopsies and observe that whenever residual CFTR-mediated $\mathrm{Cl}^{-}$secretion is present in pHNEs, it is also detected in rectal biopsies (Figure 2A, individuals with WT, P205S/Y1092X, and D614G/F508del genotypes). Similarly, when there is no CFTRmediated $\mathrm{Cl}^{-}$secretion in pHNEs, it is also not detected in rectal biopsies (Figure 2A, individuals with F508del/F508del, N1303K/F508del, and G85E/1717-1G >A genotypes). In fact, there is a significant correlation of $95 \%(p$-value $=0.001)$ between data obtained in these two models (Figure 2B).

\subsection{Rescue of CFTR-Mediated $\mathrm{Cl}^{-}$Secretion by CFTR Modulators $p H N E s$ Correlate with CFTR Responses Measured in Intestinal Organoids}

Since rectal biopsies cannot be directly used to assess the effect of CFTR modulators, a recent model of intestinal organoids that undergo forskolin-induced swelling (FIS) was developed. In this assay, the swelling of the organoids is directly dependent of CFTR function $[18,20]$. Thus, we also compared CFTR rescue by CFTR modulators in pHNEs and in intestinal organoids. To this end, intestinal organoids and $\mathrm{pHNEs}$ were incubated with CFTR correctors VX-809 or VX-661 for $24 \mathrm{~h}$ and $48 \mathrm{~h}$, respectively, for each cellular model.

Our results show that, as expected, incubation with VX-809 or VX-661 resulted in CFTR rescue in both the intestinal organoids and pHNEs of PwCF homozygous for F508del (Figure $3 \mathrm{~A}-\mathrm{C}$ ). In contrast, no CFTR rescue was observed in the intestinal organoids or pHNEs of the individual bearing the G85E/1717-1G>A genotype (Figure 3D-F). A clear CFTR rescue by CFTR modulators was observed in both the intestinal organoids and pHNEs of the PwCF bearing the P205S mutation (Figure 3G-I, $p$-value $<0.01$ ) when nontreated and treated cells are compared. Although CFTR rescue is clear when analyzing the intestinal organoids of the individual with the D614G mutation at lower concentrations 
of Fsk, such as $0.128 \mu \mathrm{M}$ (Figure 3J-L), this effect was not significant when comparing non-treated and treated pHNEs from the same individual (Figure $3 \mathrm{H}$ ).

Nasal Cells

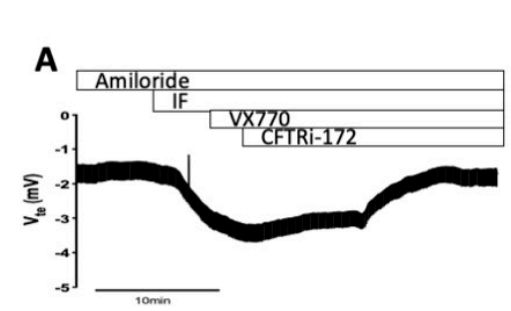

\section{Rectal Biopsies}

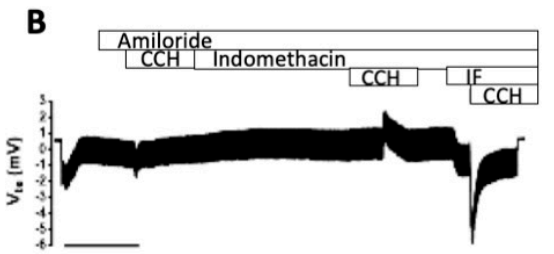

2. F508del/F508del
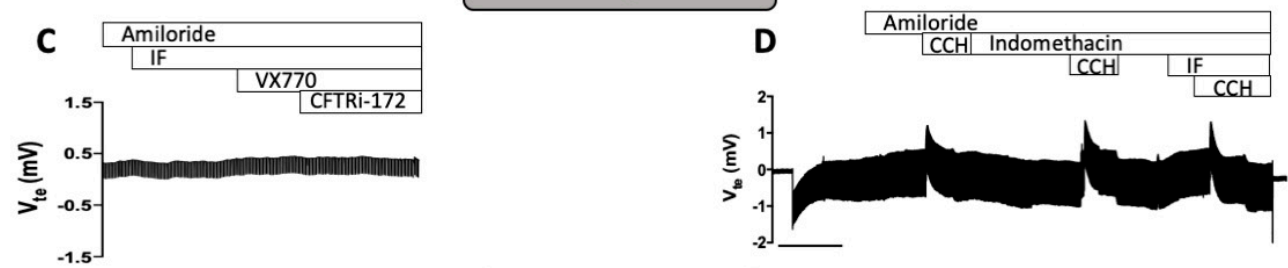

3. P205S/Y1092X
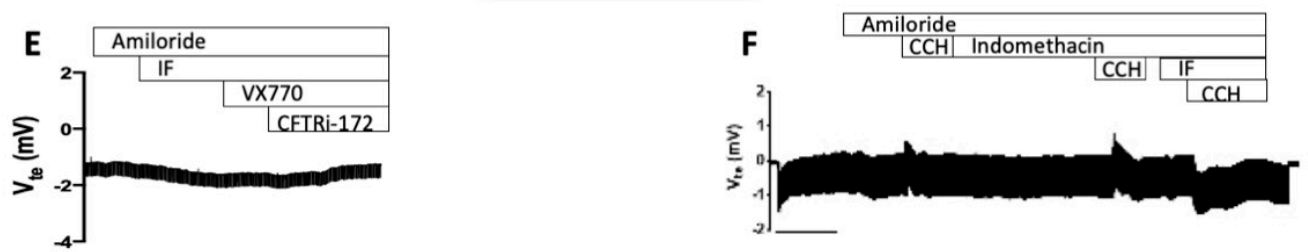

4. D614G/F508del
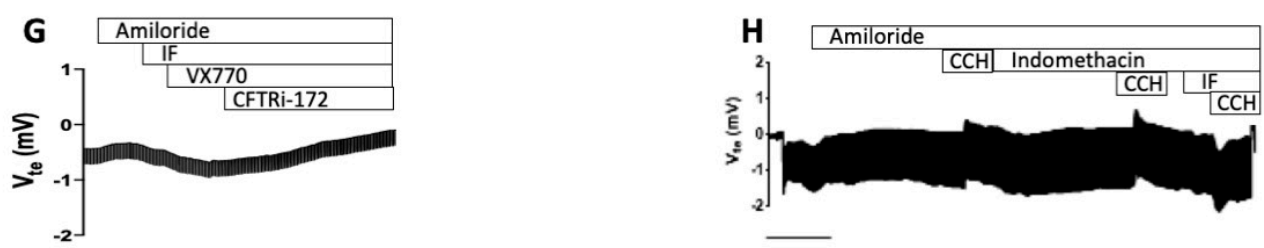

\section{N1303K/F508de}
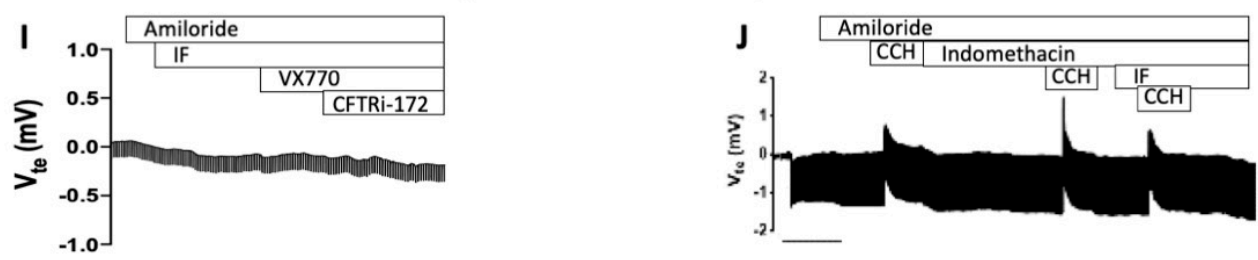

\section{G85E/1717-1G>A}
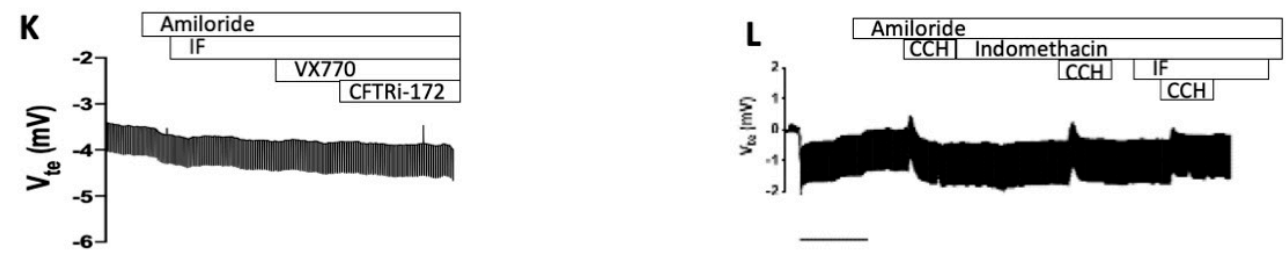

Figure 1. Comparison of basal CFTR-mediated $\mathrm{Cl}^{-}$secretion in primary human nasal epithelial cells (pHNEs) and rectal biopsies. Measurements of basal CFTR-mediated $\mathrm{Cl}^{-}$secretion in pHNEs (left panels) in samples from one healthy control 
and five PwCF with different genotypes show similarities with the basal CFTR-mediated $\mathrm{Cl}^{-}$secretion measured in rectal biopsies (right panels) from the same individual. The sample code and genotypes are indicated above the tracings. (A,C,E,G,I,K) Original Ussing chamber (open-circuit) recordings showing transepithelial voltage measurements (Vte) obtained for pHNE monolayers with different CFTR genotypes, after cAMP-dependent activation. Amiloride (20 $\mu$ M) was kept during the whole experiment duration to avoid interference of ENaC-mediated currents. Lumen-negative transepithelial Vte deflections are observed following luminal stimulation by forskolin alone (Fsk, $2 \mu \mathrm{M})$ or together with potentiator ivacaftor $(\mathrm{VX770}, 3 \mu \mathrm{M})$. The latter is fully reverted by application of the specific CFTR inhibitor CFTRInh$172(30 \mu \mathrm{M})$. (B,D,F,H,J,L) Representative original recordings of the effects of cholinergic (by carbachol $(\mathrm{CCH}), 100 \mu \mathrm{M}$, basolateral) and cAMP-dependent (by IBMX/Fsk (I/F), $100 \mu \mathrm{M} / 2 \mu \mathrm{M}$, basolateral) $\mathrm{Cl}^{-}$secretion on transepithelial Vte in rectal biopsies.
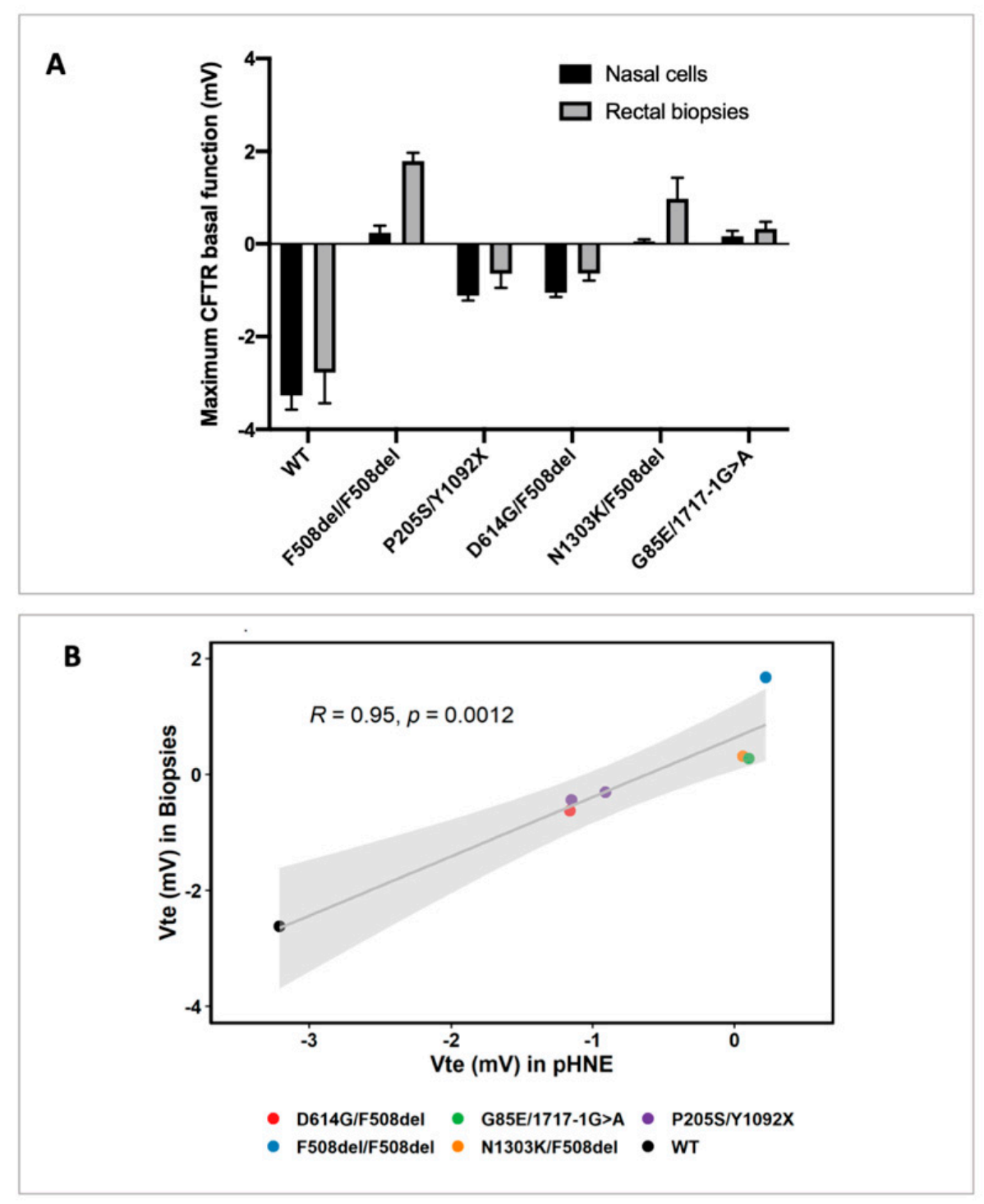

Figure 2. Correlation between maximum activation of CFTR-mediated $\mathrm{Cl}^{-}$secretion in pHNEs and rectal biopsies from the same individual. (A) Summary of CFTR-mediated $\mathrm{Cl}^{-}$secretion in rectal biopsies and pHNE cells in one healthy control and five different individuals with CF. CFTR basal function in pHNEs correspond to the differences in Vte after IBMX/Fsk stimulation. CFTR basal function in rectal biopsies is expressed by the difference in Vte upon IBMX/Fsk+IBMX/Fsk/CCH stimulation. Data is represented as the mean of three different experiments for each model \pm SD. (B) Correlation of CFTR-mediated $\mathrm{Cl}^{-}$secretion stimulated by IBMX/Fsk in pHNEs and IBMX/Fsk+ IBMX/Fsk/CCH in rectal biopsies $\left(\mathrm{Vte}_{\mathrm{I} / \mathrm{F}+\mathrm{I} / \mathrm{F} / \mathrm{C}}\right)$ from the same individual (Pearson's $\mathrm{R}=0-95, p$-value $\left.=0.0012\right)$. The grey line represents the linear regression of the data. The grey area represents the $95 \%$ confidence interval. 
Intestinal Organoids

\section{Nasal Cells}

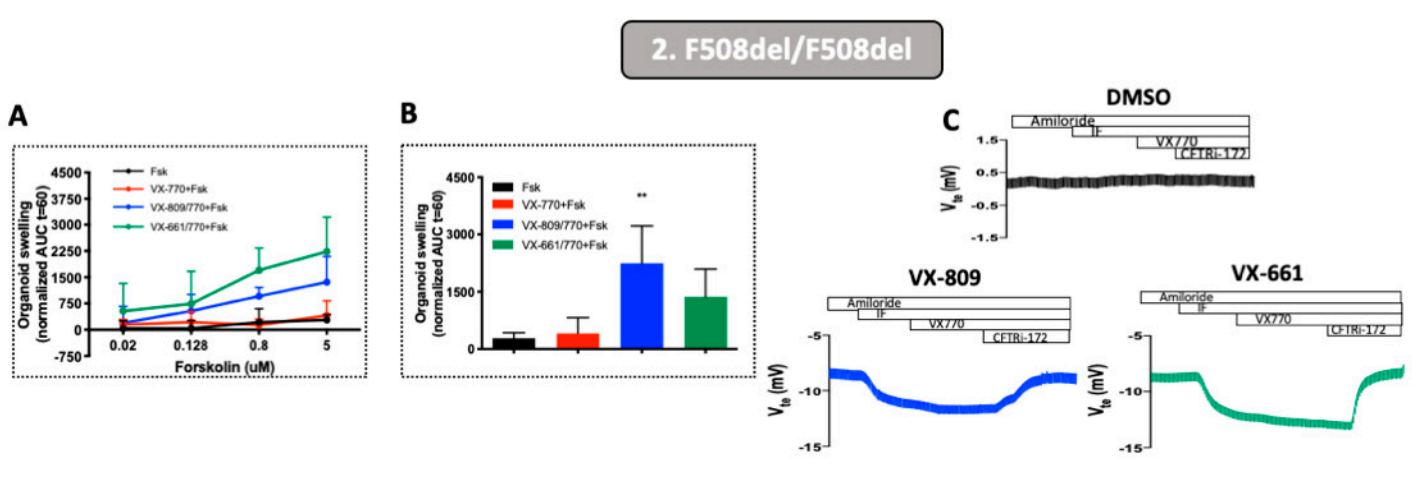

\section{G85E/1717-1G>A}

D

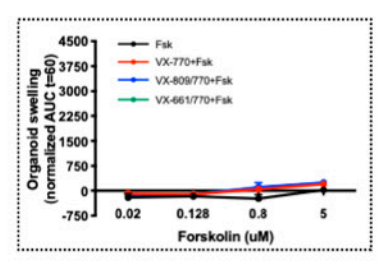

E

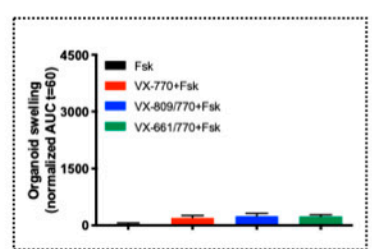

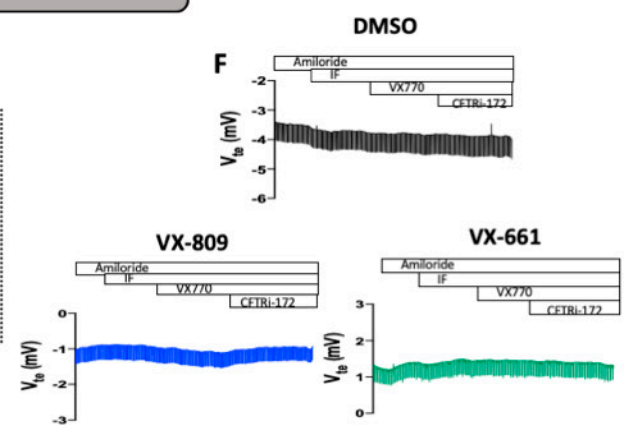

3. P205S/Y1092X

G

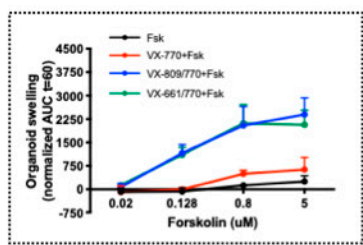

H

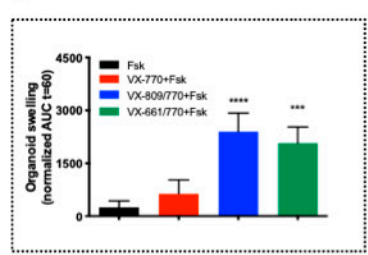

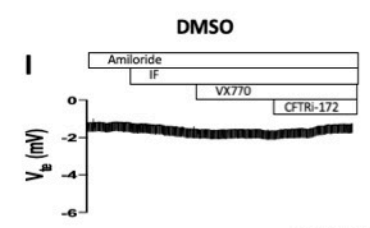

\section{D614G/F508del}

J

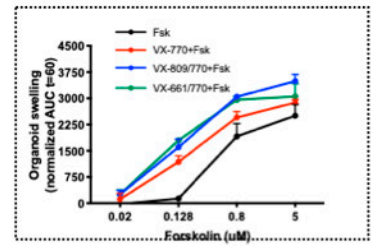

K

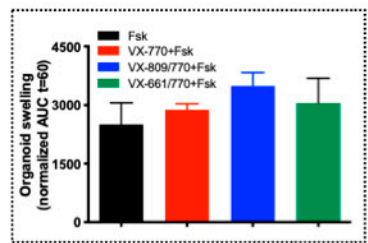

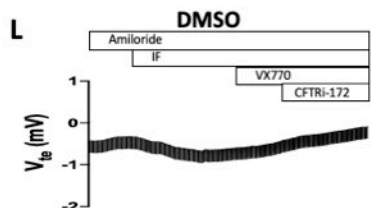
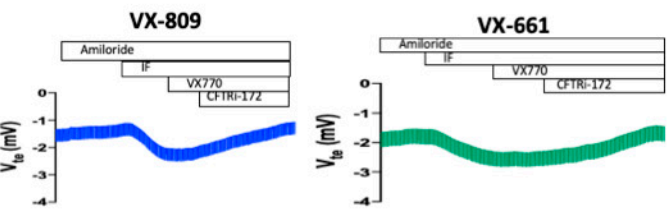

Figure 3. Comparison of CFTR rescue by CFTR modulators in pHNEs and intestinal organoids. Measurements of CFTR rescue by CFTR modulators show similar rescue in $\mathrm{pHNEs}$ and intestinal organoids from the same individual. Quantification 
of forskolin-induced swelling (FIS) assay in intestinal organoids from (A) PwCF homozygous for F508del (control, individual 2); PwCF with the (D) G85E/1717-1G>A (individual 6); (G) P205S/Y1092X (individual 3); and (J) D614G/F508del (individual 4) genotypes treated with ivacaftor (VX-770, $3 \mu \mathrm{M})$, lumacaftor (VX-809, $3 \mu \mathrm{M})$, and tezacaftor (VX-661, $5 \mu \mathrm{M})$ at forskolin (Fsk) concentrations of $0.02,0.128,0.8$, and $5 \mu \mathrm{M}$. FIS data are expressed as the area under the curve (AUC) of organoid surface area increase (baseline $=100 \%, t=60 \mathrm{~min}$ ). Quantification of these is shown in graphs $(\mathbf{B}, \mathbf{E}, \mathbf{H}, \mathbf{K})$ Data represent the mean of measurements \pm SD. $p$-value is indicated in the figure $\left(* *=<0.01 ;{ }^{* * *}=<0.001 ;{ }^{* * *}=<0.0001\right)$; original Ussing chamber (open-circuit) recordings showing CFTR-mediated $\mathrm{Cl}^{-}$secretion measured as transepithelial voltage (Vte) obtained for $\mathrm{pHNE}$ monolayers from the same individual (C,F,I,L), also treated with DMSO, $3 \mu \mathrm{M}$ of VX-809 or $5 \mu \mathrm{M}$ VX-661 for $48 \mathrm{~h}$. Data is represented as the mean of three different technical replicates for each model.

Next, we performed a comparative analysis of CFTR rescue by CFTR modulators in pHNEs and intestinal organoids. We observe that when we detect CFTR rescue in pHNEs, we also detect it in intestinal organoids, and when there is no rescue of CFTR function in pHNEs, we were also unable to detect it in intestinal organoids (Figure 3, black bars vs. color bars, respectively). Indeed, regarding CFTR rescue by VX-661, when the organoids of PwCF positively respond in a FIS assay (AUC > 1000), the pHNEs of PwCF also display CFTR rescue (Figure 4A). The same is observed for VX-809 (Figure 4B). Finally, the results show that there is a significant correlation of $88-91 \%(p$-value $=0.03)$ between these two models (Figure 4C,D).
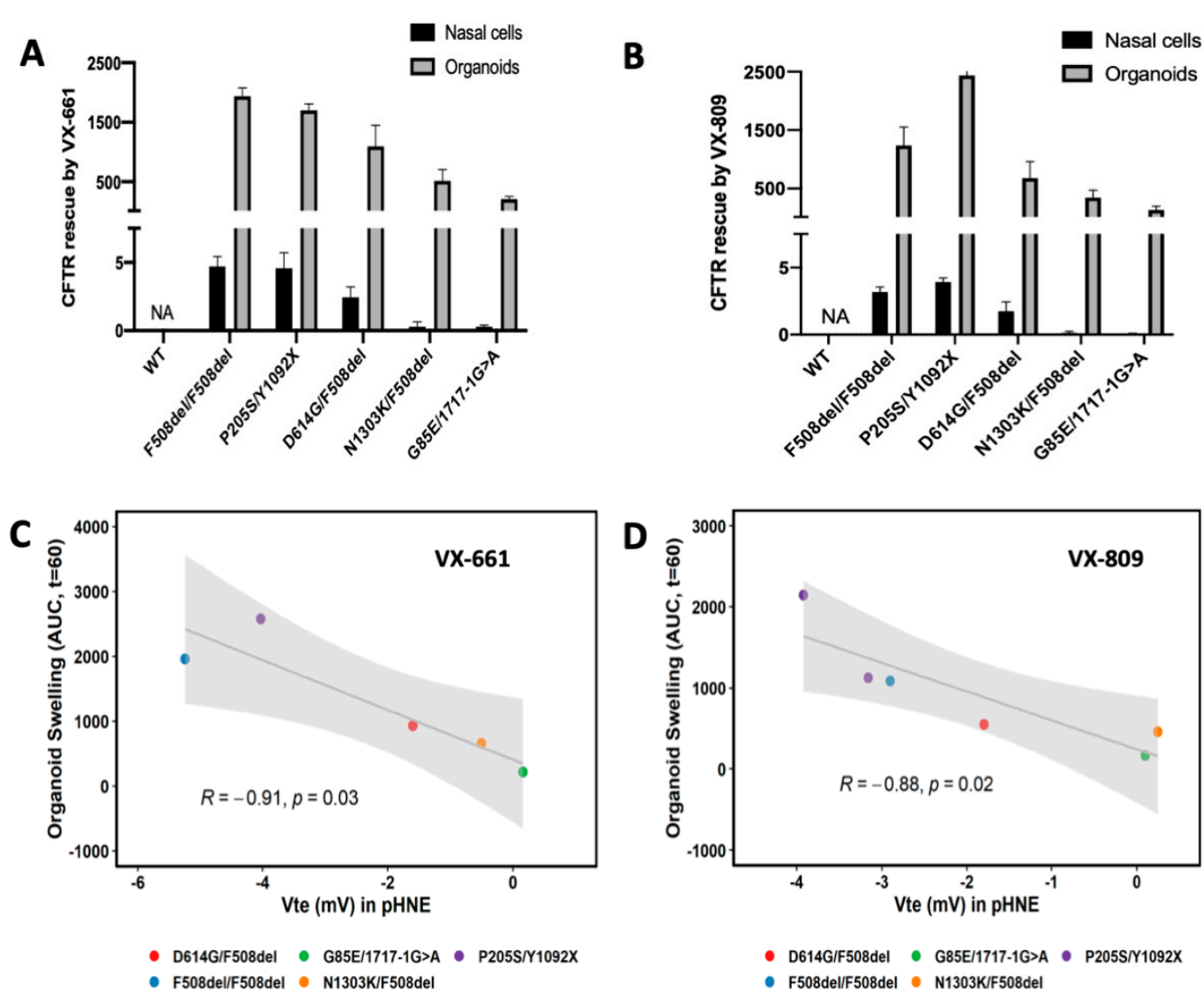

Figure 4. Correlation between CFTR rescue by CFTR modulators in pHNEs and intestinal organoids. (A,B) Summary of responses in intestinal organoids (shown as $\triangle \mathrm{AUC}$ (DMSO-treated) $t=60,3 \mu \mathrm{M}$ VX-809 or $5 \mu \mathrm{M}$ VX-661) and pHNE cells (shown as transepithelial voltage (Vte)) in five different individuals. WT function in intestinal organoids is not comparable due to pre-swelling (due to CFTR pre-activation by endogenous cAMP). According to standard protocols, pHNEs were treated with DMSO, $3 \mu \mathrm{M}$ VX-809, or $5 \mu \mathrm{M}$ VX-661 for $48 \mathrm{~h}$ and intestinal organoids for $24 \mathrm{~h}$. Data is represented as the mean of three technical replicates for each model \pm SD. (C,D) Correlation analysis of CFTR rescue in pHNEs and intestinal organoids from the same individual (Pearson's $\mathrm{R}=-0.91, p$-value $=0.03$ for VX-661 and Pearson's $\mathrm{R}=-0.88, p$-value $=0.02$ for VX-809). The grey line represents the linear regression of the data. The grey area represents the $95 \%$ confidence interval. 


\section{Discussion}

As highly effective CFTR modulator therapies (HEMT) emerge, there is an unmet need to find effective drugs for individuals with $\mathrm{CF}$ with ultra-rare mutations, for whom there are no dedicated drug discovery programs [3,40-44]. Therefore, the development of techniques and models that reliably predict the clinical benefit from CFTR modulator therapies are required for these individuals through personalized approaches termed theranostics [41]. Here, we assess CFTR basal function and the individual responses to CFTR modulators in primary human nasal epithelial ( $\mathrm{pHNE}$ ) cells from PwCF carrying rare mutations and compare these measurements with those in native rectal biopsies and intestinal organoids.

Our results show a significant and high correlation (95\%) between CFTR basal function measured in pHNEs and rectal biopsies (Figure 2). This was somewhat expected since measurements of CFTR-mediated $\mathrm{Cl}^{-}$secretion in both pHNEs and rectal biopsies have already been published by several independent groups and show good correlations with clinical data $[16,20,31,33,40]$. Additionally, CFTR activation in these two models has some practical similarities since CFTR is activated by the same concentration of Fsk and IBMX ( $2 \mu \mathrm{M}$ and $100 \mu \mathrm{M}$, respectively) and CFTR activity is measured by the same electrophysiological technique, i.e., in the Ussing chamber. It is important to note that the positive values measured in rectal biopsies, shown in Figure 2A, reflect the cholinergic effect of $\mathrm{CCH}$, which gives rise to luminal positive responses as described by others $[30,32,33,45]$ due to the activation of potassium channels and the lack of CFTR function to compensate for this ion transport.

Here, we also found a significant and high correlation (91\%) between CFTR rescue by CFTR modulators in pHNEs and intestinal organoids (Figure 4). This finding highlights that both cellular models can be used to predict the clinical benefit of a single drug or combination of drugs for a certain individual, despite the fact that CFTR function in pHNEs was measured by an Ussing chamber, whereas in intestinal organoids, it was indirectly measured by organoid swelling. Several groups already use either of these two models to predict responses to CFTR modulator drugs [16-18,20,39,40,46-49], but here, we show that the establishment of a responder or non-responder was the same in both models (Figure 3), using both types of samples from the same individual. The only exception was the individual with the D614G/F508del genotype who was considered a non-responder through pHNE analysis but appeared as a responder from intestinal organoid data. This was probably because D614G is a mutation associated with residual CFTR activity and usage of $2 \mu \mathrm{M}$ of Fsk in pHNEs already over-stimulated CFTR (Figure 3H). This is confirmed by the intestinal organoids results that show at $0.128 \mu \mathrm{M}$ of Fsk (Figure 3G) a clear difference between the control (black line at $0.128 \mu \mathrm{M}$ ) and the treatments (red, blue, and green lines); however, at $0.8 \mu \mathrm{M}$ Fsk, that is no longer a significant difference due to high residual function (Figure 3G, black line at $0.8 \mathrm{M}$ ). This also stresses the fact that a series of Fsk dilutions is able to better distinguish responders from non-responders.

Regarding the sample collection, we can say that pHNEs are certainly easier to obtain (through nasal brushings) than rectal biopsies and we show that they can reflect the same CFTR activity as rectal biopsies or intestinal organoids. Moreover, other group showed that $\mathrm{pHNE}$ are also a surrogate for pHBE [50]. However, there is still a lack of standardized protocols to culture and differentiate these cells and there is a high variability among experimental replicates, even for cells from the same individual cultivated in the same lab. Nevertheless, taken together, these results show that pHNEs from different PwCF with different genotypes can be used to analyze CFTR function and rescue by modulators, in particular for those with rare mutations who cannot undergo clinical trials.

\section{Conclusions}

To conclude, our results show that pHNEs are a bona fide theranostic model to assess CFTR rescue by CFTR modulator drugs, in particular for PwCF bearing rare mutations. Moreover, the assessment of CFTR rescue by modulators should be performed using an 
individual and personalized approach, where each individual's cells are incubated with the different modulators available and CFTR rescue should be measured using different techniques that could complement each other (e.g., electrophysiological measurements in an Ussing chamber and FIS assay), aiming at predicting the best treatment that will translate into real clinical benefit.

Author Contributions: Conceptualization, I.A.L.S. and M.D.A.; methodology, I.A.L.S. and V.R.; formal analysis, I.A.L.S. and A.D.; resources, M.D.A.; data curation, I.A.L.S.; writing-original draft preparation, I.A.L.S. and V.R.; writing—review and editing, M.D.A.; supervision, M.D.A.; funding acquisition, M.D.A. All authors have read and agreed to the published version of the manuscript.

Funding: Work was supported by the UIDB/04046/2020 and UIDP/04046/2020 center grants (to BioISI) and research grants (to MDA); "iDrugCF" (FCT/02/SAICT/2017/28800), both from FCT/MCTES Portugal; “HIT-CF” (H2020-SC1-2017-755021) from EU; SRC 013 from CF Trust-United Kingdom; "PTSense" (AMARAL19G0) from CFF-United States; and Donation Grant from Vertex Pharmaceuticals for CFTR genotyping.

Institutional Review Board Statement: The study was conducted according to the guidelines of the Declaration of Helsinki and approved by the Ethics Committee of the Santa Maria Hospital and D. Estefânia Hospital (Lisboa, Portugal).

Informed Consent Statement: Informed consent was obtained from all subjects involved in the study.

Data Availability Statement: Data available on request due to space restrictions. The raw data presented in this study are available on request from the corresponding author. The data are not publicly available due to space restrictions.

Acknowledgments: The authors would like to acknowledge the Microscopy Facility at the Faculty of Sciences of the University of Lisboa (MF-FCUL). MF-FCUL is a node of the Portuguese Platform of BioImaging, reference PPBI-POCI-01-0145-FEDER-022122. The authors are also grateful to all individuals with CF and healthy volunteers that kindly agree to participate in this study and to all the doctors involved in this work, namely José Cavaco, Susana Castanhinha, Celeste Barreto, Telma Barbosa, among others, for their important collaboration.

Conflicts of Interest: The authors declare no conflict of interest.

$\begin{array}{ll}\text { Abbreviations } \\ \text { AUC } & \text { area under the curve } \\ \mathrm{CCH} & \text { Carbachol } \\ \mathrm{CF} & \text { Cystic Fibrosis } \\ \mathrm{CFTR} & \text { Cystic Fibrosis Transmembrane Conductance Regulator } \\ \mathrm{Cl}^{-} & \text {chloride } \\ \mathrm{ENaC}^{-} & \text {Epithelial Sodium Channel } \\ \text { Fsk } & \text { forskolin } \\ \mathrm{FIS} & \text { forskolin induced swelling assay } \\ \mathrm{HCO} & - \\ \mathrm{IBMX}^{-} & \text {bicarbonate } \\ \text { pHNE } & \text { 3-Isobutyl-1-methylxanthine } \\ \text { pHBE } & \text { primary human nasal epithelial cells } \\ \mathrm{PwCF} & \text { people with CF } \\ \text { Vte } & \text { Voltage }\end{array}$

\section{References}

1. De Boeck, K.; Amaral, M.D. Progress in therapies for cystic fibrosis. Lancet Respir. 2016, 2600, 1-13. [CrossRef]

2. Elborn, J.S.; Davies, J.; Mall, M.A.; Flume, P.A.; Plant, B. Current strategies for the long-term assessment, monitoring, and management of cystic fi brosis patients treated with CFTR modulator therapy. J. Cyst. Fibros. 2016, 16, 11-12.

3. Lopes-Pacheco, M. CFTR Modulators: The Changing Face of Cystic Fibrosis in the Era of Precision Medicine. Front. Pharmacol. 2020, 10, 1662. [CrossRef] [PubMed]

4. Cuthbertson, L.; Walker, A.W.; Oliver, A.E.; Rogers, G.B.; Rivett, D.W.; Hampton, T.H.; Ashare, A.; Elborn, J.S.; De Soyza, A.; Carroll, M.P.; et al. Lung function and microbiota diversity in cystic fibrosis. Microbiome 2020, 8, 1-13. [CrossRef] 
5. Cantin, A.M.; Hartl, D.; Konstan, M.W.; Chmiel, J.F. Inflammation in cystic fibrosis lung disease: Pathogenesis and therapy. J. Cyst. Fibros. 2015, 14, 419-430. [CrossRef]

6. Blanchard, A.; Waters, V. Microbiology of Cystic Fibrosis Airway Disease. Semin. Respir. Crit. Care Med. 2019, 40, 727-736. [CrossRef]

7. Cystic Fibrosis Mutation Database. Available online: http://www.genet.sickkids.on.ca (accessed on 20 April 2021).

8. Middleton, P.G.; Hospital, W.; Mall, M.A.; Republic, C.; Lands, L.C.; Medicine, P.R.; Cystic, P.; Clinic, F.; Mckone, E.F.; Polineni, D.; et al. Elexacaftor-Tezacaftor-Ivacaftor for Cystic Fibrosis with a Single Phe508del Allele. N. Engl. J. Med. 2019, 381, 1809-1819. [CrossRef]

9. Pranke, I.; Golec, A.; Hinzpeter, A.; Edelman, A.; Sermet-Gaudelus, I. Emerging therapeutic approaches for cystic fibrosis. From gene editing to personalized medicine. Front. Pharmacol. 2019, 10, 1-21. [CrossRef]

10. Ridley, K.; Condren, M. Elexacaftor-tezacaftor-ivacaftor: The first triple-combination cystic fibrosis transmembrane conductance regulator modulating therapy. J. Pediatr. Pharmacol. Ther. 2020, 25, 192-197. [CrossRef]

11. Harutyunyan, M.; Huang, Y.; Mun, F.; Yang, F.; Arora, K.; Naren, A. Personalized medicine in CF: From modulator development to therapy for cystic fibrosis patients with rare CFTR mutations. Am. J. Physiol. Lung. Cell Mol. Physiol. 2018, 314, L529-L543. [CrossRef] [PubMed]

12. Skov, M.; Hansen, C.R.; Pressler, T. Cystic fibrosis-An example of personalized and precision medicine. Apmis 2019, 127, 352-360. [CrossRef] [PubMed]

13. Elborn, S.; Vallieres, E. Cystic fibrosis gene mutations: Evaluation and assessment of disease severity. Adv. Genom. Genet. 2014, 4, 161. [CrossRef]

14. Mou, H.; Brazauskas, K.; Rajagopal, J. Personalized medicine for cystic fibrosis: Establishing human model systems. Pediatr. Pulmonol. 2015, 50, S14-S23. [CrossRef]

15. Marson, F.A.L.; Bertuzzo, C.S.; Ribeiro, J.D. Personalized or precision medicine? The example of cystic fibrosis. Front. Pharmacol. 2017, 8, 390. [CrossRef] [PubMed]

16. Awatade, N.T.; Uliyakina, I.; Farinha, C.M.; Clarke, L.A.; Mendes, K.; Solé, A.; Pastor, J.; Margarida, M.; Amaral, M.D. Measurements of Functional Responses in Human Primary Lung Cells as a Basis for Personalized Therapy for Cystic Fibrosis. EBioMedicine 2015, 2, 147-153. [CrossRef]

17. Awatade, N.T.; Ramalho, S.; Silva, I.A.L.; Felício, V.; Botelho, H.M.; de Poel, E.; Vonk, A.; Beekman, J.M.; Farinha, C.M.; Amaral, M.D. R560S: A class II CFTR mutation that is not rescued by current modulators. J. Cyst. Fibros. 2019, 18, 182-189. [CrossRef] [PubMed]

18. Dekkers, J.F.; Berkers, G.; Kruisselbrink, E.; Vonk, A.; De Jonge, H.R.; Janssens, H.M.; Bronsveld, I.; Van De Graaf, E.A.; Nieuwenhuis, E.E.S.; Houwen, R.H.J.; et al. Characterizing responses to CFTR-modulating drugs using rectal organoids derived from subjects with cystic fibrosis. Sci. Transl. Med. 2016, 8, 344ra84. [CrossRef]

19. Veit, G.; Xu, H.; Dreano, E.; Avramescu, R.G.; Bagdany, M.; Beitel, L.K.; Roldan, A.; Hancock, M.A.; Lay, C.; Li, W.; et al. Structure-guided combination therapy to potently improve the function of mutant CFTRs. Nat. Med. 2018, 24, 1732-1742. [CrossRef]

20. Silva, I.A.L.; Doušová, T.; Ramalho, S.; Centeio, R.; Clarke, L.A.; Railean, V.; Botelho, H.M.; Holubová, A.; Valášková, I.; Yeh, J.T.; et al. Organoids as a personalized medicine tool for ultra-rare mutations in cystic fibrosis The case of S955P and 1717-2A > G. Biochim. Biophys. Acta-Mol. Basis Dis. 2020, 1866, 165905. [CrossRef]

21. Laselva, O.; Eckford, P.D.; Bartlett, C.; Ouyang, H.; Gunawardena, T.N.; Gonska, T.; Moraes, T.J.; Bear, C.E. Functional rescue of c.3846G $>$ A (W1282X) in patient-derived nasal cultures achieved by inhibition of nonsense mediated decay and protein modulators with complementary mechanisms of action. J. Cyst. Fibros. 2020, 19, 717-727. [CrossRef]

22. Laselva, O.; Moraes, T.J.; He, G.; Bartlett, C.; Szàrics, I.; Ouyang, H.; Gunawardena, T.N.A.; Strug, L.; Bear, C.E.; Gonska, T. The cftr mutation c. $3453 \mathrm{~g}>\mathrm{c}(\mathrm{d} 1152 \mathrm{~h})$ confers an anion selectivity defect in primary airway tissue that can be rescued by ivacaftor. $J$. Pers. Med. 2020, 10. [CrossRef]

23. Wu, Y.S.; Jiang, J.; Ahmadi, S.; Lew, A.; Laselva, O.; Xia, S.; Bartlett, C.; Ip, W.; Wellhauser, L.; Ouyang, H.; et al. ORKAMBImediated rescue of mucociliary clearance in cystic fibrosis primary respiratory cultures is enhanced by arginine uptake, arginase inhibition, and promotion of nitric oxide signaling to the cystic fibrosis transmembrane conductance regulator channe. Mol. Pharmacol. 2019, 96, 515-525. [CrossRef]

24. Amato, F.; Scudieri, P.; Musante, I.; Tomati, V.; Caci, E.; Comegna, M.; Maietta, S.; Manzoni, F.; Di Lullo, A.M.; De Wachter, E.; et al. Two CFTR mutations within codon 970 differently impact on the chloride channel functionality. Hum. Mutat. 2019, 40, 742-748. [CrossRef]

25. de Winter-De Groot, K.M.; Janssens, H.M.; van Uum, R.T.; Dekkers, J.F.; Berkers, G.; Vonk, A.; Kruisselbrink, E.; Oppelaar, H.; Vries, R.; Clevers, H.; et al. Stratifying infants with cystic fibrosis for disease severity using intestinal organoid swelling as a biomarker of CFTR function. Eur. Respir. J. 2018, 52. [CrossRef]

26. Scudieri, P.; Musante, I.; Venturini, A.; Guidone, D.; Genovese, M.; Cresta, F.; Caci, E.; Palleschi, A.; Poeta, M.; Santamaria, F.; et al. Ionocytes and CFTR Chloride Channel Expression in Normal and Cystic Fibrosis Nasal and Bronchial Epithelial Cells. Cells 2020, 9, 1-15. [CrossRef] [PubMed] 
27. Pedemonte, N.; Bertozzi, F.; Caci, E.; Sorana, F.; Di Fruscia, P.; Tomati, V.; Ferrera, L.; Rodríguez-Gimeno, A.; Berti, F.; Pesce, E.; et al. Discovery of a picomolar potency pharmacological corrector of the mutant CFTR chloride channel. Sci. Adv. 2020, 6, 1-13. [CrossRef]

28. Randell, S.; Fulcher, M.; O'Neal, W.; Olsen, J. Primary epithelial cell models for cystic fibrosis research. Methods Mol. Biol. 2011, 742, 285-310. [PubMed]

29. Van Mourik, P.; Beekman, J.M.; Van Der Ent, C.K. Intestinal organoids to model cystic fibrosis. Eur. Respir. J. 2019, 54, 1-6. [CrossRef] [PubMed]

30. Silva, I.A.L.; Duarte, A.; Marson, F.A.L.; Centeio, R.; Doušová, T.; Kunzelmann, K.; Amaral, M.D. Assessment of Distinct Electrophysiological Parameters in Rectal Biopsies for the Choice of the Best Diagnosis/Prognosis Biomarkers for Cystic Fibrosis. Front. Physiol. 2020, 11, 1-12. [CrossRef]

31. Mall, M.; Hirtz, S.; Gonska, T.; Kunzelmann, K. Assessment of CFTR function in rectal biopsies for the diagnosis of cystic fibrosis. J. Cyst. Fibros. 2004, 3, 165-169. [CrossRef]

32. Hirtz, S.; Gonska, T.; Seydewitz, H.H.; Thomas, J.; Greiner, P.; Kuehr, J.; Brandis, M.; Eichler, I.; Rocha, H.; Lopes, A.I.; et al. CFTR Cl-Channel Function in Native Human Colon Correlates With the Genotype and Phenotype in Cystic Fibrosis. Gastroenterology 2004, 127, 1085-1095. [CrossRef]

33. Sousa, M.; Servidoni, M.F.; Vinagre, A.M.; Ramalho, A.S.; Ribeiro, M.A.; Uliyakina, I.; Marson, F.A.; Bonadia, L.C.; Ribeiro, D.; Bertuzzo, C.S.; et al. Measurements of CFTR-Mediated Cl 2 Secretion in Human Rectal Biopsies Constitute a Robust Biomarker for Cystic Fibrosis Diagnosis and Prognosis. PLoS ONE 2012, 7, e47708. [CrossRef] [PubMed]

34. Ratjen, F.; Döring, G. Cystic fibrosis. Lancet 2003, 361, 681-689. [CrossRef]

35. Servidoni, M.F.; Sousa, M.; Vinagre, A.M.; Cardoso, S.R.; Ribeiro, M.A.; Meirelles, L.R.; de Carvalho, R.B.; Kunzelmann, K.; Ribeiro, A.F.; Ribeiro, J.D.; et al. Rectal forceps biopsy procedure in cystic fibrosis: Technical aspects and patients perspective for clinical trials feasibility. BMC Gastroenterol. 2013, 13, 8-11. [CrossRef] [PubMed]

36. Vonk, A.M.; van Mourik, P.; Ramalho, A.S.; Silva, I.A.L.; Statia, M.; Kruisselbrink, E.; Suen, S.W.F.; Dekkers, J.F.; Vleggaar, F.P.; Houwen, R.H.J.; et al. Protocol for Application, Standardization and Validation of the Forskolin-Induced Swelling Assay in Cystic Fibrosis Human Colon Organoids. STAR Protoc. 2020, 1, 100019. [CrossRef] [PubMed]

37. Harris, C.M.; Mendes, F.; Dragomir, A.; Doull, I.J.M.; Carvalho-Oliveira, I.; Bebok, Z.; Clancy, J.P.; Eubanks, V.; Sorscher, E.J.; Roomans, G.M.; et al. Assessment of CFTR localisation in native airway epithelial cells obtained by nasal brushing. J. Cyst. Fibros. 2004, 3, 43-48. [CrossRef]

38. Hagemeijer, M.; Vonk, A.; Awatade, N.; Silva, I.; Tischer, C.; Hilsenstein, V.; Beekman, J.; Amaral, M.; Botelho, H. An open-source high-content analysis workflow for CFTR function measurements using the forskolin-induced swelling assay. Bioinformatics 2020, btaa1073. [CrossRef]

39. Ramalho, A.S.; Förstová, E.; Vonk, A.M.; Ferrante, M.; Verfailli, C.; Dupont, L.; Boon, M.; Proesmans, M.; Beekma, J.M.; Sarouk, I.; et al. Correction of CFTR function in intestinal organoids to guide treatment of cystic fibrosis. Eur. Respir. J. 2021, 57, 1902426. [CrossRef]

40. Laselva, O.; McCormack, J.; Bartlett, C.; Ip, W.; Gunawardena, T.N.A.; Ouyang, H.; Eckford, P.D.W.; Gonska, T.; Moraes, T.J.; Bear, C.E. Preclinical studies of a rare cf-causing mutation in the second nucleotide binding domain (C.3700a $>$ g) show robust functional rescue in primary nasal cultures by novel cftr modulators. J. Pers. Med. 2020, 10, 1-11. [CrossRef]

41. Amaral, M.D. How to determine the mechanism of action of CFTR modulator compounds: A gateway to theranostics. Eur. J. Med. Chem. 2021, 210, 112989. [CrossRef]

42. Molinski, S.V.; Ahmadi, S.; Ip, W.; Ouyang, H.; Villella, A.; Miller, J.P.; Lee, P.; Kulleperuma, K.; Du, K.; Di Paola, M.; et al. Orkambi ${ }^{\circledR}$ and amplifier co-therapy improves function from a rare CFTR mutation in gene-edited cells and patient tissue. $E M B O$ Mol. Med. 2017, 9, 1224-1243. [CrossRef]

43. Kato, A.; Romero, M.F. Regulation of Electroneutral $\mathrm{NaCl}$ Absorption by the Small Intestine. Annu. Rev. Physiol. 2011. [CrossRef]

44. Phuan, P.W.; Haggie, P.M.; Tan, J.A.; Rivera, A.A.; Finkbeiner, W.E.; Nielson, D.W.; Thomas, M.M.; Janahi, I.A.; Verkman, A.S. CFTR modulator therapy for cystic fibrosis caused by the rare c.3700A $>$ G mutation. J. Cyst. Fibros. 2020, S1569-1993, 1-8.

45. Roth, E.K.; Hirtz, S.; Duerr, J.; Wenning, D.; Eichler, I.; Seydewitz, H.H.; Amaral, M.D.; Mall, M.A. The K + channel opener 1-EBIO potentiates residual function of mutant CFTR in rectal biopsies from cystic fibrosis patients. PLoS ONE 2011, 6. [CrossRef] [PubMed]

46. Berkers, G.; van Mourik, P.; Vonk, A.M.; Kruisselbrink, E.; Dekkers, J.F.; de Winter-de Groot, K.M.; Arets, H.G.M.; Marck-van der Wilt, R.E.P.; Dijkema, J.S.; Vanderschuren, M.M.; et al. Rectal Organoids Enable Personalized Treatment of Cystic Fibrosis. Cell Rep. 2019, 26, 1701-1708. [CrossRef] [PubMed]

47. Laselva, O.; Bartlett, C.; Gunawardena, T.N.A.; Ouyang, H.; Eckford, P.D.W.; Moraes, T.J.; Bear, C.E.; Gonska, T. Rescue of multiple class II CFTR mutations by elexacaftor+ tezacaftor+ivacaftor mediated in part by the dual activities of Elexacaftor as both corrector and potentiator. Eur. Respir. J. 2020, 2002774. [CrossRef]

48. Veit, G.; Roldan, A.; Hancock, M.A.; da Fonte, D.F.; Xu, H.; Hussein, M.; Frenkiel, S.; Matouk, E.; Velkov, T.; Lukacs, G.L. Allosteric folding correction of F508del and rare CFTR mutants by elexacaftor-tezacaftor-ivacaftor (Trikafta) combination. JCI Insight 2020, 5,1-14. [CrossRef] 
49. Laselva, O.; Bartlett, C.; Popa, A.; Ouyang, H.; Gunawardena, T.N.A.; Gonska, T.; Moraes, T.J.; Bear, C.E. Emerging preclinical modulators developed for F508del-CFTR have the potential to be effective for ORKAMBI resistant processing mutants. J. Cyst. Fibros. 2021, 20, 106-119. [CrossRef]

50. Brewington, J.J.; Filbrandt, E.T.; LaRosa, F.J.; Moncivaiz, J.D.; Ostmann, A.J.; Strecker, L.M.; Clancy, J.P. Brushed nasal epithelial cells are a surrogate for bronchial epithelial CFTR studies. JCI Insight 2018, 3, 1-14. [CrossRef] [PubMed] 